\section{Quelques théorèmes sur les fonctions indépendantes ${ }^{1}$ )}

par

J. MARCINKIEWICZ et A. ZYGMUND (Wilno).

\section{$\S 1$.}

Dans cette Note nous nous occupons de quelques problèmes concernant la théorie de fonctions indépendantes. Dans le $\S 2$ nous donnons des théorèmes expliquant les relations mutuelles de la théorie des fonctions indépendantes et celle des probabilités. Dans les $\S \S 3$ et 4 nous généralisons certains théorèmes démontrés dans notre travail antérieur $\left.{ }^{2}\right)$. Dans le dernier paragraphe nous établissons quelques résultats sur la convergence et la sommabilité de séries de fonctions indépendantes.

\section{$\S 2$.}

Etant donnée une suite infinie

$$
\left\{f_{n}(t)\right\}
$$

de fonctions mesurables définies dans l'intervalle $(0,1)$ il est possible de trouver une suite de fonctions indépendantes

$$
\left\{x_{n}(1)\right\}
$$

de sorte que chaque fonction $x_{n}$ soit équimesurable avec $\left.f_{n}^{y}\right)$. Ce fait est la base de l'équivalence d'une suite de variables aléatoi-

1) Sur les définitions des fonctions indépendantes voir: $M . K a c$, Sur les fonctions indépendantes (I), Studia Math. 6 (1936) p. 58-76; A. Kolmogo$r$ off, Comptes Rendus de l'Académile Communiste, 1929; p. 8-21. Comparer aussi : J. Marcinkiewicz et A. $\mathrm{Z}$ ygmund, Sur les fonctions indépendantes, Fund. Math. 29 (1937) p. 60-90.

2) Loc. cit. '), pp. 66 et 87.

3). Voir M. Ka c, loc. cit. p. 58 et notre travail cité sous ') p. 61. res indépendantes avec une certaine suite de fonctions indépendantes. Or, comme la définition de la suite (2.2) (la suite (2.1) étant donnée) n'est pas univoque, il est clair que la dite équivalence est possible seulement pour ces propriétés de fonctions indépendantes, qui sont invariantes pour toute représentation (2.2) de (2.1).

Il en résulte que les recherches de telles propriétés peuvent présenter un certain intérêt.

Nous appellerons deux suites $\left\{f_{n}\right\}$ et $\left\{g_{n}\right\}$ équimesurables ${ }^{3 a}$ ) si pour chaque $n$ les fonctions $f_{n}$ et $g_{n}$ sont équimesurables.

Thé or ème 1. Soient $B_{1}, B_{2}, B_{3}, \ldots, B_{n}, \ldots$ des ensembles boreliens et $\left\{x_{n}(t)\right\}$ et $\left\{x_{n}^{\prime}(t)\right\}$ deux suites équimesurables de fonctions indépendantes. On $a$

(2.3) $\left|B_{1} x_{1} \cdot B_{2} x_{2} \cdot B_{3} x_{3} \ldots B_{n} x_{n} \ldots\right|=\left|B_{1} x_{1}^{\prime} \cdot B_{2} x_{2}^{\prime} \cdot B_{3} x_{3}^{\prime} \ldots B_{n} x_{n}^{\prime} \ldots\right|$, où $\quad B_{n} x_{n}=E_{t}\left(x_{n}(t) \in B_{n}\right)$ et $B_{n} x_{n}^{\prime}=E_{t}\left(x_{n}^{\prime}(t) \in B_{n}\right) \quad(n=1,2, \ldots)$.

La démonstration de ce théorème est immédiate. En effet, les fonctions $x_{n}$ et $x_{n}^{\prime}$ étant mesurables et les ensembles $B_{n}$ boreliens, les ensembles $B_{n} x_{n}$ et $B_{n} x_{n}^{\prime}$ sont mesurables. D'autre part, les fonctions $x_{n}$ et $x_{n}^{\prime}$ étant équimesurables, on a

$$
\left|B_{n} x_{n}\right|=\left|B_{n} x_{n}^{\prime}\right| \text {. }
$$

D'après l'indépendance des fonctions $\left\{x_{n}\right\}$ on conclut (2.5) $\left|B_{1} x_{1} . B_{2} x_{2} . B_{3} x_{3} \ldots B_{n} x_{n} \ldots\right|=\left|B_{1} x_{1}\right| .\left|B_{2} x_{2}\right| \ldots\left|B_{n} x_{n}\right| \ldots$

et de même

(2.6) $\left|B_{1} x_{1}^{\prime}, B_{2} x_{2}^{\prime}, B_{3} x_{3}^{\prime} \ldots B_{n} x_{n}^{\prime} \ldots\right|=\left|B_{1} x_{1}^{\prime}\right| .\left|B_{2} x_{2}^{\prime}\right| \ldots\left|B_{n} x_{n}^{\prime}\right| \ldots$

Les égalités (2.4), (2.5) et (2.6) donnent (2.3).

Nous appellerons une fonction $f\left(x_{1}, x_{2}, \ldots, x_{n}, \ldots\right)$ borelienne, s'il existe une suite de fonctions continues $\left\{f_{n}\left(x_{1}, x_{2}, \ldots, x_{n}\right)\right\}$ telle que pour toute suite $x_{1}, x_{2}, \ldots, x_{n}, \ldots$ on a

$$
\lim _{n \rightarrow \infty} f_{n}\left(x_{1}, x_{2}, \ldots, x_{n}\right)=f\left(x_{1}, x_{2}, \ldots, x_{n}, \ldots\right) .
$$

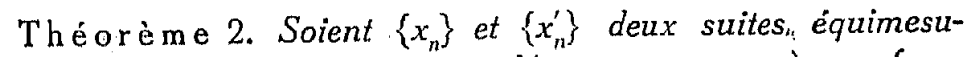
rables de fonctions indépendantes et $f\left(x_{1}, x_{2}, \ldots, x_{n}, \ldots\right)$ une fonc-

39) MM. Kac et Steinhaus (Studia Math. 6 (1936) p. 93) employent le mot: „équivalentes". 
tion borelienne. Les fonctions $F(t)=f\left(x_{1}(t), x_{2}(t), \ldots, x_{n}(t), \ldots\right)$ et $F^{\prime}(t)=f\left(x_{1}^{\prime}(t), x_{2}^{\prime}(t), \ldots, x_{n}^{\prime}(t), \ldots\right)$, considérées comme fonctions de la variable $t$, sont équimesurables.

Démonstration. Soit $f_{n}(x)=f_{n}\left(x_{1}, x_{2}, \ldots x_{n}\right)$ une suite de fonctions continues satisfaisant à la condition (2.7). Il suffit évidemment de démontrer que les fonctions

$F_{n}(t)=f_{n}\left(x_{1}(t), x_{2}(t), \ldots, x_{n}(t)\right)$ et $F_{n}^{\prime}(t)=f_{n}\left(x_{1}^{\prime}(t), x_{2}^{\prime}(t), \ldots, x_{n}^{\prime}(t)\right)$ sont équimesurables. Or, la fonction $f_{n}(x)$ étant continue, l'ensemble $n$-dimensionnel

$$
\underset{\left(x_{1}, x_{2}, \ldots x_{n}\right)}{E\left\{\left(f_{n}\left(x_{1}, x_{2}, \ldots x_{n}\right)<a\right)\right\}}
$$

est ouvert, il est donc une somme d'une suite de cubes disjoints, disons $K_{1}, K_{2}, \ldots, K_{n}, \ldots$ Posons

$E_{n}=E_{t}\left[\left(x_{1}(l), x_{2}(l), \ldots x_{n}(t)\right) \in K_{m}\right], E_{m}^{\prime}=E_{t}\left[\left(x_{1}^{\prime}(1), x_{2}^{\prime}(l), \ldots x_{n}^{\prime}(t)\right) \in K_{m}\right]$.

Les ensembles $E_{m}$ étant disjoints, il suffit de démontrer les égalités

$$
\left|E_{m}\right|=\left|E_{m}^{\prime}\right| \quad(m=1,2, \ldots) \text {. }
$$

Supposons par exemple que $K_{m}$ soit défini par les relations

$$
a_{1} \leqslant x_{1} \leqslant b_{1}, a_{2} \leqslant x_{2} \leqslant b_{2}, \ldots, a_{n} \leqslant x_{n} \leqslant b_{n} .
$$

Les fonctions $x_{n}$ étant indépendantes, la mesure de l'ensemble $E_{m}$ est égale au produit des mesures des ensembles

$$
E\left(a_{r} \leqslant x_{r} \leqslant b_{r}\right) .
$$

Il en est de même aussi pour l'ensemble $E_{m}^{\prime}$. Or, comme

$$
\left|E\left(a_{v} \leqslant x_{r} \leqslant b_{v}\right)\right|=\left|E\left(a_{v} \leqslant x_{r}^{\prime} \leqslant b_{v}\right)\right|,
$$

on en obtient la proposition demandée.

\section{Théorème 3. Soient}

$$
\left\{f_{n}(x)\right\}=\left\{f_{n}\left(x_{1}, x_{2}, \ldots, x_{n}\right)\right\}
$$

une suite de fonctions continues, $x_{n}(t)$ et $x_{n}^{\prime}(t)$ deux suites équimesurables de fonctions indépendantes. Les mesures des ensembles de convergence des suites sont égales.

$$
\begin{gathered}
F_{n}(t)=f_{n}\left(x_{1}(t), x_{2}(t), \ldots x_{n}(t)\right) \\
F_{n}^{\prime}(t)=f_{n}\left(x_{1}^{\prime}(t), x_{2}^{\prime}(t), \ldots, x_{n}^{\prime}(t)\right)
\end{gathered}
$$

Démonstration. Posons pour $n \leqslant m$

$$
D_{n m}(x)=\max _{n \leq p, q \leq m}\left|f_{p}\left(x_{1}, x_{2}, \ldots, x_{p}\right)-f_{q}\left(x_{1}, x_{2}, \ldots, x_{n}\right)\right|
$$

Les fonctions $\Phi_{n m}(x)$ sont continues, les fonctions

$\Phi_{n m}(t)=\Phi_{n m}\left(x_{1}(t), x_{2}(t), \ldots, x_{m}(t)\right)$ et $\Phi_{n m}^{\prime}(1)=\Phi_{n m}\left(x_{1}^{\prime}(t), x_{2}^{\prime}(t), \ldots, x_{n}^{\prime}(t)\right)$

sont donc équimesurables. On en conclut que les fonctions

$$
W_{n}(t)=\lim _{m \rightarrow \infty} \Phi_{n m}(t) \text { et } \phi_{n}^{\prime}(t)=\lim _{m \rightarrow \infty} \phi_{n m}^{\prime}(t)
$$

sont aussi équimesurables. Le même subsiste aussi pour les fonctions

$$
\Phi(t)=\lim _{n \rightarrow \infty} \Phi_{n}(t), \quad \Phi^{\prime}(t)=\lim _{n \rightarrow \infty} \Phi_{n}^{\prime}(t) .
$$

Les ensembles de convergence des suites (2.8) étant caractérisés par les relations

$$
\Phi(t)=0 \text { respectivement } \Phi^{\prime}(t)=0,
$$

ils sont de la même mesure.

Thé or è me 4. Soit donnée une suite $f_{n}(x)=f_{n}\left(x_{1}, x_{2}, \ldots x_{n}\right)$ de fonctions continues. Supposons que les suites

$\left\{f_{n}\left(a_{1}, a_{2}, \ldots a_{p-1}, a_{p}, a_{p+1}, \ldots, a_{n}\right)\right\}$ et $\left\{f_{n}\left(a_{1}, a_{2}, \ldots a_{p-1}, b_{p}, a_{p+1}, \ldots, a_{n}\right)\right\}$ sont équiconvergentes $\left.{ }^{4}\right)$ quels que soient $\left\{a_{k}\right\}, b_{p}$ et $p$. Alors la suite

$$
\left\{F_{n}(t)\right\}=\left\{f_{n}\left(x_{1}(t), x_{2}(t), \ldots, x_{n}(t)\right)\right\}
$$

converge presque partout, ou bien elle ne converge que dans un ensemble de mesure nulle $\left.{ }^{4 a}\right)$.

Démonstration. Nous supposons d'abord que chaque fonction $x_{\text {n }}$ admette un ensemble tout au plus dénombrable de valeurs différentes. D'après le théorème précédent il suffit de démontrer notre proposition pour une suite $x_{n}^{\prime}(t)$ équimesu-

4) C'est-à-dire elles sont simultanément toutes les deux ou bien convergentes ou bien divergentes.

4n) Comparer A. Kolmogor off, Grundbegriffe der Wahrscheinlichkeitsrechnung, Berlin (1933) p. 60-61. 
rable avec $x_{n}(t)$ et du reste choisie d'une façon arbitraire. Soient donc $y_{n}(t)$ des fonctions indépendantes, équimesurables avec les $x_{n}(t)$ et constantes par intervalles. Posons $x_{1}^{\prime}=y_{1}$. Divisons chaque segment de constance de la fonction $x_{1}^{\prime}$ en deux parties égales, et définissons la fonction $x_{2}^{\prime}$ comme semblable ${ }^{5}$ ) à $y_{2}$ dans chaque de ces segments. Divisons ensuite chaque segment de constance de $x_{2}^{\prime}$ en deux parties égales et définissons $x_{3}^{\prime}$ comme semblable à $y_{3}$ dans chaque des segments ainsi obtenus. On définit de même les fonctions $x_{3}^{\prime}, x_{4}^{\prime}, \ldots$

Pour chaque $n$ l'intervalle $(0,1)$ se décompose en certains segments $A_{1}^{(n)}, A_{2}^{(n)}, \ldots$ tels que $\max _{k}\left|\Delta_{k}^{(n)}\right| \rightarrow 0$ et les fonctions $x_{m}^{\prime}$ pour $m>n$ sont semblables dans deux segments quelconques $A_{k}^{(n)}$ et $A_{k^{\prime}}^{(n)}$.

Il en résulte que la densité moyenne de l'ensemble $E$ de convergence de la suite $\left\{f_{n}\left(x_{1}^{\prime}(t), x_{2}^{\prime}(t), \ldots, x_{n}^{\prime}(t)\right)\right\}$, étant indépendante des valeurs de $x_{1}, x_{2}, \ldots x_{n}$, est la même dans chaque segment $A_{k}^{(n)}$; elle est donc égale à $|E|$. On en conclut que la densité inférieure de $E$ en chaque point $t$ de l'intervalle $(0,1)$ est au moins égale à $|E|$. Il en résulte, d'après le théorème classique de M. Lebesgue, que la mesure de $E$ est égale à un ou à zéro.

Nous allons maintenant considérer un autre cas spécial. Supposons notamment que toutes les fonction $x_{v}$ soient bornées, $\left|x_{v}\right| \leqslant M_{v}$, et qu'il existe une suite $k(1) \leqslant k(2) \leqslant \ldots \leqslant k(n) \rightarrow \infty$, telle que $f_{n}$ ne dépend pas des variables $x_{1}, x_{2}, \ldots, x_{k(n)}$, c'est à dire que $f_{n}=f_{n}\left(x_{k(n)+1}, \ldots, x_{n}\right)$. Posons $l_{1}=1$ et définissons l'entier $l_{i+1}>l_{i}$ par la condition $k\left(l_{i+1}\right) \geqslant l_{i}(i=1,2, \ldots)$. La variable $x_{v}$, où $l_{i-1} \leqslant \nu \leqslant l_{i}$ ne peut donc intervenir que dans les fonctions $f_{n}$, où $l_{i-1} \leqslant n \leqslant l_{i+1}$. Les fonctions $f_{n}$ étant continues, il existe un $\varepsilon_{i}>0$ tel que les inégalités

$$
\left|x_{v}-x_{v}^{\prime}\right| \leqslant \varepsilon_{i}, \quad\left|x_{v}\right| \leqslant M_{v}, \quad\left|x_{v}^{\prime}\right| \leqslant M_{v} \quad\left(l_{l-1} \leqslant v \leqslant l_{i}\right)
$$

entraînent $\left|f_{n}-f_{n}^{\prime}\right| \leqslant 1 / i$ pour $l_{i-1} \leqslant n \leqslant l_{i+1}$, où $f_{n}^{\prime}$ s'obtient de $f_{n}$ en y remplaçant $x_{p}$ par $x_{v}^{\prime}(\nu=1,2, \ldots)$. Soit $x_{v}(t)$ une suite arbitraire de fonctions indépendantes, chacune n'admettant qu'un

5) On dit qu'une fonction $f(x)$ définie dans l'intervalle $(a, b)$ et une fonction $g(x)$ définie dans l'intervalle $(c, d)$ sont semblables, si l'on a

$$
f[a+(b-a) x]=g[c+(d-c) x] \text { pour } 0 \leqslant x \leqslant 1 \text {. }
$$

nombre fini de valeurs et satisfaisant à (2.9), où $x_{v}$ et $x_{v}^{\prime}$ désignent $x_{v}(t)$ et $x_{v}^{\prime}(t)$. Comme $f_{n}-f_{n}^{\prime}$ tend alors vers zéro pour tout $t$ et comme le théorème a été déjà démontré pour les fonctions $x_{i,}^{\prime}(t)$, il subsiste pour les $x_{v}^{\prime}(t)$.

On peut se débarasser de l'hypothèse que les $x_{v}$ soient bornées, en les remplaçant par les fonctions $\bar{x}_{v}(t)$ égales à $x_{v}(t)$ si $\left|x_{v}(t)\right| \leqslant M_{v}$ et égales à zéro ailleurs. Il suffit de choisir les $M_{*}$ de sorte que $\sum_{n}\left|\underset{t}{E}\left(\left|x_{n}\right| \geqslant M_{n}\right)\right|<\infty$.

${ }_{*}$ Passons au cas general. Posons $q_{p, q}\left(x_{1}, x_{2}, \ldots, x_{q}\right) \max _{p \leq m, n \leq q}\left|f_{n}\left(x_{1}, x_{2}, \ldots, x_{n}\right)-f_{m}\left(x_{1}, x_{2}, \ldots, x_{m}\right)\right|$, où $x_{1}, x_{2}, \ldots$ sont des nombres arbitraires. Soit $E$ l'ensemble de points $t$ où la suite $\left\{f_{n}\left(x_{1}(t), \ldots, x_{n}(t)\right)\right\}$ converge. Il est facile de choisir la fonction $q=q(p)$ de sorte que l'ensemble $A$ de points $t$ où la suite $\Phi_{p, q(p)}\left(x_{1}(t), \ldots, x_{q(p)}(t)\right)$ converge vers zéro soit égal à $E+N$, où $|N|=0$. Posons $\Phi_{p, q}^{s}\left(x_{s+1}, \ldots, x_{n}\right)$ $=\Phi_{p, q}\left(0,0, \ldots, x_{s+1}, \ldots, x_{n}\right)$ et rappelons que pour tout $s$ la suite $\left\{f_{n}\left(0, \ldots, 0, x_{s-1-1}(t), \ldots, x_{n}(t)\right)\right\}$ est équiconvergente avec $\left\{f_{n}\left(x_{1}(t), \ldots, x_{n}(t)\right)\right\}$. On peut définir une suite $q=q(p)=q_{s}(p)$ de sorte que pour tout $s$ la suite $\phi_{p, q_{s}(p)}^{s}\left(x_{s+1}(t), \ldots, x_{q_{s}(p)}(t)\right)$ converge vers zéro dans un ensemble $A_{s}=E+N_{s},\left|N_{s}\right|=0$. En multipliant $q_{p, \eta_{s}(p)}^{s}$ par une constante convenable $K_{s}$, on peut s'arranger que la limite supérieure de la suite $K_{s} \Phi_{p, q_{s}(p)}^{s}$ surpasse $s$ dans $C A_{s}$, sauf peut étre un ensemble de mesure $\leqslant 2^{-s}$. Il en résulte qu'il existe deux suites $q=q(p)$ et $s=s_{p} \rightarrow \infty$ telles que la suite $A_{s_{p}}()_{p, q s_{p}}^{s_{p}}(p)$ converge vers zéro presque partout dans $E$ et diverge presque partout dans $C E$. Nous nous trouvons ainsi dans un cas spécial considéré auparavant, ce qui donne $|E|=0 *$ ).

\section{§3.}

Thé o r èm e 5. Soit $\left\{x_{n}\right\}$ une suite de fonctions indépendantes à valeurs moyennes nulles. On a pour chaque $p \geqslant 1$

$$
A_{p} \int_{0}^{1}\left(\sum_{v=1}^{\infty} x_{i}^{2}\right)^{p / 2} d t \leqslant \int_{0}^{1}\left|\sum_{v=1}^{\infty} x_{v}\right|^{p} d t \leqslant B_{p} \int_{0}^{1}\left(\sum_{n=1}^{\infty} x_{v}^{2}\right)^{p / 2} d t,
$$

*") Ajouté pendant la correction des épreuves, le 8. 11. 1937. 
où $A_{p}$ et $B_{p}$ ne dépendent que de $p$. Pour $1 \leqslant p \leqslant 2$ on a les inégalités $A_{p} \geqslant A>0, B_{p} \leqslant B<\infty$, où $A$ et $B$ désignent des constantes absolues.

Les inégalités (3.1) pour $1<p<\infty$ ont été déjà démontrées dans un autre travail $\left.{ }^{6}\right)$.

Lemme 1. Si les fonctions $x$ et $x^{\prime}$ sont independantes, $x$ étant équimesurable avec $-x^{\prime}$ et si l'on a

$$
\int_{0}^{1} x d t=\int_{1}^{1} x^{\prime} d t=0
$$

alors

$$
\bar{A}_{p} \int_{i}^{1}|x|^{p} d t \leqslant \int_{0}^{1}\left|x+x^{\prime}\right|^{p} d t \leqslant \bar{B}_{p} \int_{0}^{1}|x|^{n} d t \quad(p \geqslant 1),
$$

où $\bar{A}_{p}$ et $\bar{B}_{p}$ remplissent des conditions analogues aux celles $d u$ théorème 5 .

Il suffit évidemment de démontrer la première partie de cette inégalité. Soit $0<\Delta<1$. Supposons que

$$
\int_{0}^{1}|x|^{p} d t=1 \text {. }
$$

Deux cas peuvent se produire; ou bien

$$
\int_{0}^{1}|x| d t \leqslant d
$$

ou bien

Posons

$$
\int_{0}^{1}|x| d t>4
$$

$$
A=E_{t}(x(t) \leqslant 0), \quad B=E_{t}(x(t)>0), \quad E=E_{t}(|x| \geqslant 1),
$$

et désignons par $A^{\prime}$ et $B^{\prime}$ les ensembles correspondants pour la fonction $x^{\prime}$. En supposant la validité de l'inégalité (3.5), on a

$$
\int_{C E}|x|^{p} d t \leqslant \int_{C E}|x| d t \leqslant \measuredangle
$$

") Voir notre travail cité sous 1), p. 87.
Or, d'après l'inégalité de Minkowski et (3.7), on a

$$
\begin{aligned}
& \left\{\int_{C E}\left|x+x^{\prime}\right|^{p} d t\right\}^{1 / p} \geqslant\left\{\int_{C E}\left|x^{\prime}\right|^{p} d t\right\}^{1 / p}-\left\{\int_{C E}|x|^{p} d t\right\}^{1 / p} \\
& \because\left\{|C E| \int_{0}^{1}\left|x^{\prime}\right|^{p} d t\right\}^{1 / p}-\Delta^{1 / p} \geqslant(1-\Delta)^{1 / p}-d^{1 / p}
\end{aligned}
$$

En supposant (3.6), on a d'après (3.2)

$$
\int_{A}|x| d t=\int_{B}|x| d t \geqslant \Delta / 2
$$

et, à plus forte raison,

$$
\int_{A}|x|^{p} d t=(\alpha / 2)^{p}, \quad \int_{B}|x|^{p} d t \geqslant(\Delta / 2)^{n} .
$$

Des formules analogues subsistent aussi pour la fonction $x^{\prime}$. Soit $|A| \geqslant 1 / 2$. On a

$$
\begin{gathered}
\int_{0}^{1}\left|x+x^{\prime}\right|^{p} d t \geqslant \int_{A A^{\prime}}\left|x+x^{\prime}\right|^{p} d t \geqslant \int_{A A^{\prime}}\left|x^{\prime}\right|^{p} d t \\
-=|A| \int_{A^{\prime}}\left|x^{\prime}\right|^{p} d t \geqslant 1 / 2(\Delta / 2)^{p} .
\end{gathered}
$$

En posant $f-1 / 4$, on tire de (3.8) et (3.10)

$$
\int_{0}^{1}\left|x+x^{\prime}\right|^{n} d t \geqslant \min \left[(1 / 8)^{p} / 2, \quad\left(3^{1 / p}-1\right)^{p} / 4\right],
$$

ce qui est la formule demandée.

Nous passons maintenant à la démonstration du théorème 5 .

Soient $\left\{x_{n}\right\}$ la suite donnée et $\left\{x_{n}^{\prime}\right\}$ une suite de fonctions indépendantes et équimesurables avec $\left\{-x_{n}\right\}$. Posons

$$
y_{n}=x_{n}+x_{n}^{\prime} \text {. }
$$

Désignons par $\omega_{n}(\theta)$ la suite des fonctions de RADEMACHER. Les fonctions $y_{n}$ admettent des distribuantes symétriques, donc, d'après le théorème 2, les fonctions de $t$

$$
\sum_{n=1}^{\infty}(1)(t) y_{n}(t)
$$


sont équimesurables pour chaque $\theta$. Il en résulte que

$$
\int_{0}^{1} \int_{0}^{1}\left|\sum_{n=1}^{\infty} \omega_{n}(\theta) y_{n}(t)\right|^{p} d t d \theta=\int_{0}^{1}\left|\sum_{n=1}^{\infty} y_{n}(t)\right|^{p} d t
$$

En appliquant le lemme, on trouve

$$
\begin{gathered}
\bar{A}_{p} \int_{0}^{1} \int_{0}^{1}\left|\sum_{n=1}^{\infty} \omega_{n}(\theta) x_{n}(t)\right|^{p} d t d \theta \leqslant \int_{0}^{1} \int_{0}^{1}\left|\sum_{n=1}^{\infty} \omega_{n}(\theta) y_{n}(t)\right|^{p} d t d \theta \\
\leqslant \bar{B}_{p} \int_{0}^{1} \int_{0}^{1}\left|\sum_{n=1}^{\infty} \omega_{n}(0) x_{n}(t)\right|^{p} d t d(\theta) .
\end{gathered}
$$

En tenant compte de l'inégalité de M. Khintchine ${ }^{\eta}$ ) pour $t$ fixé, on obtient

$$
A_{p} \int_{0}^{1}\left(\sum_{n=1}^{\infty} x_{n}^{2}(t)\right)^{p / 2} d t \leqslant \int_{0}^{1}\left|\sum_{n=1}^{\infty} x_{n}\right|^{p} d t \leqslant B_{p} \int_{0}^{1}\left(\sum_{n=1}^{\infty} x_{n}^{2}\right)^{p / 2} d t \text {. }
$$

Remarquons encore que l'inégalité (3.1) tombe en défaut pour $p<1$. En effet, soit $x_{1}$ une fonction égale à 1 dans un ensemble de mesure $1-1 / m$ et à $-(m-1)$ dans l'ensemble complementaire, et $x_{2}$ une fonction équimesurable avec $-x_{1}$; on a

$$
\int_{0}^{1}\left|x_{1}+x_{2}\right|^{p} d t \leqslant \frac{2}{m}[2(m-1)]^{p}, \quad \int_{0}^{1}\left(x_{1}^{2}+x_{2}^{2}\right)^{p / 2} d t \geqslant 1 .
$$

Pour $m \rightarrow \infty$, la première partie de l'inégalité (3.1) est donc en défaut quel que soit $A_{p}$. D'autre part, soit $x_{1}$ défini comme auparavant et soient $x_{2}, x_{3}, \ldots x_{n}$ des fonctions équimesurables avec $x_{1}$; on trouve facilement que $\left|\sum_{1}^{n} x_{v}\right| \geqslant n$ pour $m-1>2 n$.

\section{La somme}

$$
\sum_{1}^{n} x_{i}^{2}
$$

étant égale à $n$ dans un ensemble de mesure $(1-1 / m)^{n}$ et ne surpassant pas $n \mathrm{~m}^{2}$ dans l'ensemble complémentaire de mesure $1-(1-1 / m)^{n}$, on a

$$
\begin{aligned}
& \text { 7) Il s'agit ici de l'inégalité } \\
& A_{p} s^{p} \leqslant \int_{0}^{1}|S|^{p} d \theta \leqslant B_{n} s^{p} \text {, où } S=\Sigma c_{n} \omega_{n}(0), s^{2}=\Sigma c_{n}{ }^{2} .
\end{aligned}
$$

$$
\begin{gathered}
\int_{0}^{1}\left|\sum_{1}^{n} x_{v}\right|^{p} d t \geqslant n^{p} \\
\int_{0}^{1}\left(\sum_{1}^{n} x_{\eta}^{2}\right)^{p / 2} \leqslant n^{p / 2}(1-1 / m)^{n}+\left[1-(1-1 / m)^{n}\right]\left(n m^{2}\right)^{p / 2} .
\end{gathered}
$$

En faisant tendre $m$ vers $\infty$, on en tire, en supposant la validité de la seconde inégalité (3.1),

$$
n^{p} \leqslant B_{p} n^{p / 2}
$$

L'entier $n$ étant arbitraire, la dernière inégalité est impossible.

\section{$\$ 4$.}

Théorème 6. Soit donnée une suite $\left\{x_{n}\right\}$ de fonctions indépendantes à valeurs moyennes nulles. Posons

$$
S(t)=\sum x_{n}(t), \quad S^{*}(t)=\max _{k}\left|\sum_{1}^{k} x_{n}(t)\right|
$$

On $a$

$$
\int_{0}^{1} S^{* p} d t \leqslant A \int_{0}^{1}|S|^{p} d t \quad(1 \leqslant p \leqslant \infty)
$$

A désignant une constante absolue.

Démonstration. Ce théorème a été démontré déjà pour $p>1$ avec $A_{p}=2 p^{p} /(p-1)^{p}$ au lieu de $\left.A^{7 \mathrm{a}}\right)$. Nous pouvons donc nous borner au cas $1 \leqslant p \leqslant 2$. Il suffit évidemment de démontrer le théorème pour un nombre fini $n$ de fonctions. Nous pouvons aussi admettre que chaque fonction $x_{v}$, ne prend qu'un nombre fini de valeurs différentes.

Nous nous occuperons d'abord de fonctions à distribuantes symétriques. On a

$$
S^{*}(t)=\left|\sum_{1}^{n(t)} x_{v}(t)\right|,
$$

où $n(t)$ désigne une fonction n'admettant que de valeurs entières. Pour chaque 0 fixé, la suite $\left\{\omega_{v}(\theta) x_{v}(t)\right\}$ est équimesurable avec la suite $\left\{x_{1 \prime}(t)\right\}$. On a donc, d'après le théorème 2,

7a) Voir notre travail cité sous $\left.{ }^{1}\right)$, p. 66. 


$$
\int_{0}^{1} \int_{0}^{1}\left|\sum_{n=1}^{n(\theta, t)} \omega_{n}(\theta) x_{n}(t)\right|^{p} d t d \theta=\int_{0}^{1}\left|\sum_{1}^{n(t)} x_{v}(t)\right|^{p} d t,
$$

où $n(\theta, t)$ est définie de sorte que

$$
\left|\sum_{n=1}^{n\left\langle\theta_{,} t\right)} \omega_{\eta}(\theta) x_{\psi}(t)\right|=\max _{1 \leq k \leq n}\left|\sum_{n=1}^{k} \omega_{\eta}(0) x_{\eta}(t)\right|
$$

Or, comme le théorème est vrai pour les fonctions de Rademacher ${ }^{8}$ ), on a

$$
\int_{0}^{1}\left|\sum_{v=1}^{n(\theta, b)} \omega_{\eta}(\theta) x_{v}(t)\right|^{p} d \theta \leqslant \vec{A}\left(\sum_{1}^{n} x_{v}^{2}(t)\right)^{p / 2}
$$

En intégrant cette inégalité et en appliquant la première partie de l'inégalité (3.1), on obtient

$$
\int_{0}^{1} \int_{0}^{1}\left|\sum_{v=1}^{n(\theta, t)} \omega_{11}(0) x_{v}(t)\right|^{n} d \theta d t \leqslant A_{p}^{-1} \bar{A} \int_{0}^{1}\left|\sum_{1}^{n} x_{v}\right|^{n} d t,
$$

ou bien

$$
\int_{0}^{1} S^{* n} d t \leqslant A \int_{0}^{1}|S|^{p} d t
$$

c'est l'inégalité demandée.

Passons maintenant au cas général. Soient $x_{1}, x_{2}, \ldots x_{n}$ les fonctions indépendantes données. D'après le théorème 2, nous pouvons admettre qu'elles sont construites comme il suit: les fonctions $x_{v}$ sont constantes par intervalles et chaque fonction $x_{v}$ est semblable à elle-même dans deux segments quelconques de constance de la fonction précédente.

Soient $x_{1}^{\prime}, x_{2}^{\prime}, \ldots x_{n}^{\prime}$ les fonctions équimesurables avec $-x$ et telles que $x_{1}^{\prime}$ soit semblable à elle même dans les segments de constance de la fonction $x_{n}$, que $x_{2}^{\prime}$ soit semblable à elle même dans les segments de constance de la fonction $x_{1}^{\prime}$ et ainsi de suite. Posons

$$
x_{v}+x_{v}^{\prime}=\bar{x}_{\eta} \text {. }
$$

Les fonctions $\bar{x}_{v}$ ayant des distribuantes symétriques et étant à valeurs moyennes nulles, on a pour chaque fonction $n(t)$ n'admettant que des valeurs $1,2, \ldots n$

s) (f. notre travail cité sous '), p. 72.

$$
\int_{0}^{1}\left|\sum_{1}^{n(t)} \bar{x}_{v}(t)\right|^{p} d t \leqslant \bar{A} \int_{0}^{1}\left|\sum_{1}^{n} \bar{x}_{v}(t)\right|^{p} d t
$$

ou bien

$$
\int_{0}^{1}\left|\sum_{1}^{n(t)} \bar{x}_{v}(t)\right|^{p} d t \leqslant 2^{p} \bar{A} \int_{0}^{1}\left|\sum_{1}^{n} x_{v}(t)\right|^{p} d t .
$$

Or il est evident qu'il est possible de définir la fonction $n(t)$ de sorte qu'elle soit constante dans chaque segment de constance de la fonction $x_{n}$ et qu'elle satisfasse en outre à la condition

$$
\left|\sum_{1}^{n(t)} x_{\eta \prime}(t)\right|=\max _{1 \leq k \leq n}\left|\sum_{1}^{k} x_{\eta^{\prime}}(t)\right|
$$

En désignant par $d$ un segment quelconque où la fonction $x_{n}$ reste constante, on a

$$
\left(\int_{d}\left|\sum_{1}^{n(t)} x_{v}(t)\right|^{p} d t\right)^{1 / p} \leqslant\left(\int\left|\sum_{1}^{n(t)} \bar{x}_{v}(t)\right|^{p} d t\right)^{1 / p}+\left(\int\left|\sum_{1}^{n(t)} x_{v}^{\prime}(t)\right|^{p} d t\right)^{1 / p} .
$$

Le dernier terme à droite est égal à

$$
\left(\Delta \int_{0}^{1}\left|\sum_{1}^{m} x_{1}(t)\right|^{p} d t\right)^{1 / p} \quad(m=m(\Delta)<n)
$$

une expression qui ne surpasse pas, d'après le théorème 5 ,

$$
\left(C \Delta \int_{0}^{1}\left|\sum_{1}^{n} x_{1}(t)\right|^{p} d t\right)^{1 / p}
$$

où $C$ désigne une constante. Il en résulte que

$$
\int_{d}\left|\sum_{1}^{n(t)} x_{\eta}(t)\right|^{p} d t \leqslant 2^{p} \int_{\mathcal{J}}\left|\sum_{1}^{n(t)} \bar{x}_{\eta}(t)\right|^{p} d t+2^{p} C \Delta \int_{0}^{1}\left|\sum_{1}^{n} x_{\eta}(t)\right|^{p} d t .
$$

En faisant la somme de ces inégalités pour tous les segments $\Delta$ et en appliquant l'inégalité (4.3), on obtient d'après l'inégalité (4.4) la formule (4.2). 


\section{$\S 5$.}

Soit donné un tableau infini $(T)$ de nombres $a_{i k}$,

$$
(T)=\left(\begin{array}{llll}
a_{11}, & a_{12}, & a_{13}, & \ldots \\
a_{21}, & a_{22}, & a_{23}, & \ldots \\
a_{31}, & a_{32}, & a_{33}, & \ldots \\
\ldots & \ldots & \ldots & \ldots
\end{array}\right)
$$

tel que

$$
\lim _{i \rightarrow \infty} a_{i k}=1 ; \quad k==1,2,3, \ldots
$$

On dit qu'une série

$$
\sum_{i=1}^{\infty} u_{t}
$$

est sommable $(T)$ vers la somme $s$, si l'on a

$$
\lim _{i \rightarrow \infty} \sum_{k} a_{i k} u_{k}=S \text {, }
$$

où toutes les séries dans le membre gauche sont supposées convergentes. Nous allons démontrer le

Thé or ème 7. Étant donnée une série

$$
\sum_{n=1}^{\infty} x_{n}
$$

de fonctions indépendantes, sommable $(T)$ presque partout, on peut définir une suite numérique $\left\{\lambda_{n}\right\}$ de sorte que la série

$$
\sum_{n=1}^{\infty}\left(x_{\eta}-\lambda_{n}\right)
$$

converge presque partout.

Démonstration. Supposons d'abord les fonctions $x_{n}$ à distribuantes symétriques. En tenant compte du théorème 3 on démontre facilement que chaque série

$$
\sum_{n=1}^{\infty} \omega_{v}(\theta) x_{v}(t)
$$

est sommable $(T)$ au presque tout point $t$, c'est à dire cette série considérée comme la série de deux variables est sommable $(T)$ presque partout dans le carré $(0,1)(0,1)$. Il en résulte, d'après le théorème de Fubins, que pour presque tout $t$ la série

$$
\sum_{v=1}^{\infty} \omega_{v}(\theta) x_{v}(t)
$$

est sommable $(T)$ presque partout. Il en résulte ${ }^{9}$ ) presque partout

$$
\sum_{n=1}^{\infty} x_{\eta}^{2}(t)<\infty
$$

Supposons maintenant $x_{1}, x_{2}, \ldots$ quelconques. Les fonctions $x_{1}, x_{1}^{\prime}, x_{2}, x_{2}^{\prime}, \ldots x_{n}, x_{n}^{\prime}, \ldots$ soient indépendantes et telles que $x_{v}^{\prime}$ soit équimesurable avec $-x_{v}$. Posons $\bar{x}_{v}=x_{v}+x_{v}^{\prime}$. La série

$$
\sum_{n=1}^{\infty} \bar{x}_{n}
$$

est sommable presque partout $(T)$, ce qui donne, en vertu de (5.4),

$$
\sum_{n=1}^{\infty} \bar{x}_{n}^{2}<\infty
$$

\section{Posons}

$$
E_{v}=E_{l}\left(\left|\bar{x}_{v}\right| \geqslant 1\right), \quad A_{v}=E\left(x_{v}-\lambda_{v}>1\right), \quad B_{v}=E\left(x_{v}^{\prime}+\lambda_{v} \geqslant 0\right),
$$

où $\lambda_{y}$ est défini de sorte que l'on ait

$$
\left|E\left(x_{r} \leqslant \lambda_{\eta}\right)\right| \geqslant 1 / 2, \quad\left|E\left(x_{p} \geqslant \lambda_{v}\right)\right| \geqslant 1 / 2 .
$$

Il est evident que

$$
A_{v} B_{v} \subset E_{v}
$$

Or, comme la série

$$
\sum_{n=1}^{\infty}\left|E_{n}\right|
$$

converge, on a aussi

$$
\sum_{n=1}^{\infty}\left|A_{v} B_{v}\right|=\sum_{v=1}^{\infty}\left|A_{v}\right| .\left|B_{v}\right| \leqslant \sum_{v=1}^{\infty}\left|E_{v}\right|<\infty .
$$

Par conséquent, en vertu de (5.7) on a

$$
\sum_{v=1}^{\infty}\left|A_{v}\right|<\infty
$$

v) Ce théorème, facile à démontrer, se trouve dans une forme un peu différente chez A. Zy g mund, Fund. Math. 16 (1930) p. 100. 
En posant

$$
A_{v}^{\prime}=E\left(x_{r}-\lambda_{1}<1\right)
$$

on obtient d'une façon analogue

$$
\sum\left|A_{v}^{\prime}\right|<\infty \text {. }
$$

En vertu de (5.8) et (5.9), nous pouvons admettre que

$$
\left|x_{r}-\lambda_{v}\right| \leqslant 1 ; \quad \nu=1,2, \ldots
$$

Les fonctions $\vec{x}_{r}$ sont donc uniformément bornées. D'autre part, d'après (5.6), la série

$$
\sum_{r=1}^{\infty} \omega_{p}(t) \bar{x}_{i}(t)
$$

converge au presque tout point $(0, t)$. Il s'en suit que pour un certain point $\theta_{0}$ la série

$$
\sum_{r=1}^{\infty} \omega_{v}\left(\theta_{0}\right) \bar{x}_{v}(t)
$$

converge presque partout en $t$. Il en résulte la convergence presque partout de la série

$$
\sum_{r=1}^{\infty} \bar{x}_{r}
$$

Les fonctions $\bar{x}_{r}$ étant uniformément bornées, on a ${ }^{10}$ )

$$
\sum_{n=1}^{\infty} \int_{0}^{1} \bar{x}_{i}^{2} d t<\infty
$$

\section{Posons}

$$
\Delta_{v}=\int_{i}^{1} x_{v} d t
$$

On a

$$
\int_{0}^{1} \bar{x}_{v}^{2} d t=2 \int_{u}^{1}\left(x_{v}-\Delta_{v}\right)^{2} d t .
$$

En tenant compte de (5.12), on a

$$
\sum_{r=1}^{\infty} \int_{0}^{1}\left(x_{v}-\Delta_{\eta}\right)^{2} d t<\infty .
$$

${ }^{10)}$ A. Kolmogoroff, Math. Ann. 99 (1928) p. 314.
De la dernière formule résulte, en vertu du théorème de MM. KHINTCHINE et Kolmogorof ${ }^{11}$ ), la convergence presque partout de la série

$$
\sum x_{1} \text {. }
$$

Remarque. Le théorème 7 reste vrai si l'on suppose seulement que la série (5.2) soit fini $(T)$ presque partout, ou du moins dans un ensemble de mesure positive. La démonstration reste la même.

Théorème 8. Si la série de fonctions indépendantes

$$
\sum_{v=1}^{\infty} x_{v}
$$

converge en mesure, elle converge presque partout au sens ordinaire ${ }^{110}$.

Démonstration. La suite

$$
\left\{\sum_{1}^{n} x_{i}\right\}
$$

étant convergente en mesure, on peut en extraire une suite partielle

$$
\left\{\sum_{1}^{n} x_{n}\right\}
$$

convergente presque partout. La série considérée est donc sommable par une méthode linéaire presque partout. D'après le théorème 7 , il en résulte qu'il existe une suite numérique $\left\{i_{v}\right\}$ telle que la série

$$
\sum_{i=1}^{\infty}\left(x_{1}-\lambda_{1}\right)
$$

convergep resque partout. D'autre part, comme la série $\sum_{n=1}^{\infty} x_{n}$ converge en mesure, la série

$$
\sum_{n=1}^{\infty} \lambda_{v^{\prime}}
$$

converge. Il en résulte la convergence presque partout de la série

$$
\sum_{i=1}^{\infty} x_{i}
$$

ii) Loc. cit. ${ }^{10 \%}$.

11ii) Ce théorème se trouve aussi démontré dans le livre récent de M. P. Lévy, Variables aléatoires, Paris (1937), p. XVII +328 , spéc. p. 139. (Remarque ajoutée pendant la correction des épreuves). 
En appliquant ce théorème, on obtient le

Théorème 9. Si l'on a

$$
\lim _{m, n \rightarrow \infty} \int_{0}^{1}\left|S_{n}-S_{m}\right|^{p} d t=0 \quad(0<p<\infty)
$$

où

la série

$$
S_{n}=\sum_{1}^{n} x_{v}
$$

$$
\sum_{n=1}^{\infty} x_{n^{\prime}}
$$

converge presque partout.

Pour $1 \leqslant p \leqslant \infty$ ce théorème peut être déduit facilement $\mathrm{du}$ théorème 6 . Enfin on peut formuler le théorème 8 en termes. de fonctions caractéristiques. A savoir, on peut énoncer le théorème suivant:

Théor ème 10. Soit donnée une série

$$
\sum_{v=1}^{\infty} x_{v}
$$

de fonctions indépendantes. Soit

$$
\varphi_{r^{\prime}}(\theta)=\int_{0}^{1} e^{i \theta x_{w_{r}}(t)} d t
$$

la fonction caractéristique de $x_{\eta}$. Pour que la série (5.13) soit convergente presque partout, il faut et il suffit que le produit infini

$$
\prod_{v=1}^{\infty} \varphi_{n}(\theta)
$$

soit uniformément convergent dans tout intervalle fini.

C'est une conséquene simple du théorème 8 et d'une proposition connue de M. P. LÉvY ${ }^{12}$ ).

12) P. Lévy, Calcul des probabilités, p. 195-197. Cf. aussi M. Ka c et H. Steinhaus, Studia Math. 6 (1936) pp. 59-66.

(Reçu par la Rédaction le 14. 7. 1937). 